

\title{
Homophily in Social Media and News Polarization*
}

\author{
Luis Abreu ${ }^{\dagger} \quad$ Doh-Shin Jeon ${ }^{\ddagger}$
}

May 27, 2021

\begin{abstract}
We consider an ad-financed media firm that chooses the ideological location of its news and targets consumers who can share the news with their followers on social media. After studying how each targeted consumer's incentive to share the news is shaped by the location of the news and the distribution of her followers' ideological locations, we study the firm's strategy to maximize the breadth of news sharing and find that when the mean (respectively, the variance) of the followers' ideological locations is a convex (respectively, concave) function of a targeted consumer's location, the firm is likely to produce polarized news.
\end{abstract}

Keywords: Media Bias, Social Media, Homophily, News Sharing, Polarization

${ }^{*}$ We thank Heski Bar-Issac, Ron Berman, Francis Bloch, Giacomo Calzolari, Alexandre de Cornière, Oliver Falck, Renato Gomes, Grace Gu, Emeric Henry, Nicholas Economides, Sarit Markovich, Leslie Marx, Alessandro Pavan, Andrew Rhodes, Marcella Scrimitore, Bruno Strulovici, Freek Van Gils, Jidong Zhou and those who attended our presentation at the University of Geneva, Net Institute Conference, the 46th EARIE, Hitotsubashi University, Osaka University, TSE Seminar, the 17th Northwestern/TSE Conference on Industrial Organization, European University Institute, the 3rd Doctoral Workshop on the Economics of Digitization, the 17th IIOC, the 4th Economics of Media Bias Workshop, the 12th Digital Economics Conference and the 16th Media Economics Workshop. Both authors acknowledge financial support from the European Research Council (ERC) under the European Union's Horizon 2020 research and innovation programme (grant agreement No 670494), from ANR under grant ANR-17-EURE-0010 (Investissements d'Avenir program), and from the NET Institute (www.NETinst.org).

${ }^{\dagger}$ Toulouse School of Economics, University of Toulouse Capitole, Toulouse, France, luis.martins-abreu@utcapitole.fr

¥Toulouse School of Economics, University of Toulouse Capitole, CEPR, dohshin.jeon@tse-fr.eu 
"What information consumes is rather obvious: it consumes the attention of its recipients. Hence a wealth of information creates a poverty of attention..."

(Simon, 1971)

\section{Introduction}

Social media has become a very important source for news consumption. According to Matsa and Shearer (2018), as of August 2018, two-thirds of Americans report that they consume some news on social media - with two in ten doing so often. Among different social media, Facebook remains the most relevant: $45 \%$ of Americans consume news on Facebook.

This success of social media, however, is viewed with suspicion as some fear that the tendency whereby social media users end up mainly consuming like-minded news would prevent them from digesting diverse viewpoints about important issues. Although people express such concerns by pointing out problems such as filter bubbles (Pariser, 2011) or echo chambers (Sunstein, 2017), one might think that homophily or social network structure is a more fundamental source of the problem. Indeed, by analyzing data from US Facebook users, Bakshy, Messing and Adamic (2015) found that homophily is the most important factor limiting their exposure to attitude-challenging content. ${ }^{1}$ Even if there is a consensus that homophily on social media leads to more consumption of like-minded news, there is mixed evidence about whether social media leads to political polarization; Barberá (2015) and Boxell, Gentzkow and Shapiro (2017) find evidence inconsistent with polarization, while Allcott et al. (2020), Levi (2021), and Yanagizawa-Drott, Petrova and Enikolopov (2019) find evidence consistent with polarization.

This paper contributes to the debate on the relationship between social media and news polarization by focusing on the supply of news and addresses the following questions. How does the structure of social networks on social media affect news sharing and thereby shape the incentive for a media firm to choose the ideological bias of its news? Under what conditions is the media firm incentivized to provide polarized news? Indeed, Kümpel, Karnowski and Keyling (2015), who review 109 papers on news sharing on social media published by

\footnotetext{
${ }^{1}$ Similarly, Halberstam and Knight (2016) analyzed information from 2.2 million Twitter users on the day before the 2012 US general elections and found that, due to homophily, people are disproportionately exposed to tweets from like-minded others.
} 
communication or computer scientists, call for a theory emphasizing the role of the social networks of followers on the decision to share news. This is exactly what we attempt to accomplish.

To answer the above questions, we consider a three-tiered hierarchy: a media firm, direct consumers and their followers on social media. The media firm sends its news to targeted direct consumers, who in turn decide whether to share the news with their followers. We show that the incentive for a direct consumer to share a given news item depends on the ideological location of the news as well as the mean and the variance of the locations of her followers, which in turn depend on the direct consumer's own ideological position. Therefore, to understand news polarization, it is important to know how extremists' followers differ from those of moderates in terms of the mean and the variance of their locations. In fact, it is known that ideologically more extreme individuals tend to be more homophilous than more moderate ones (Boutyline and Willer, 2017). As our main result, we find that when the mean (respectively, the variance) of followers' ideological locations is a convex (respectively, concave) function of a direct consumer's location, the media firm is likely to produce polarized news. In contrast, when the mean is concave or the variance is convex, it is likely to produce unbiased news.

Our questions are motivated by anecdotal evidence from the US presidential election in 2016. Over one hundred websites with false content were created by teenagers from Macedonia, seeking advertising revenues propelled by sharing of their news on Facebook (Silverman and Alexander, 2016). The teenagers were using Facebook to drive traffic to their websites where they had ads from Google. Their sites produced misleading partisan content and obtained more engagement than op-eds and commentary pieces from major media (NPR, 2016). Even though our model does not make distinction between fake news and true news, our results help to understand when polarized news is more likely to be shared than less polarized news.

In Section 2, we present our baseline model, in which consumers are distributed over an interval $[0,1]$ of ideological space and a media firm chooses the ideological location of its news to maximize its advertising revenue, which is proportionate to the measure of readers. In addition, the media firm chooses an interval of given length to target direct consumers. We assume that the utility from reading the news has a quadratic loss. We adopt an altruistic motive for news sharing (Boyd, Golder and Lotan, 2010; Small, 2011; Holton et al., 2014) and 
assume that a targeted consumer shares the news if it is relevant enough to her followers, i.e., if the average benefit of her followers from reading the news is larger than the attention tax she imposes on them by sharing the news. The attention tax captures the opportunity cost of attention, as sharing news with a friend induces the latter to pay attention to it, which does not necessarily mean that the latter reads it. Using Simon's (1971) expression, we assume that sharing news "consumes the attention of its recipients". In a world of information overload, the attention tax can be high.

In Section 3, we consider no targeting in the sense that the target interval is equal to $[0,1]$. For simplicity, we assume that the constant utility from reading the news is large enough that all consumers who have access to the news read it. Under this assumption, profit maximization is equivalent to maximizing the measure of the direct consumers who share the news, which we define as the breadth of news sharing. If the attention tax is low enough, by locating the news at the middle, the media firm can induce all direct consumers to share the news. Hence, we focus on the case in which the attention tax is not small. As an important intermediary result, we show that the average benefit that followers obtain from a shared news item can be decomposed into a constant, minus the variance of their locations, minus a square of the difference between the news location and the mean of their locations. As a main result, we find that news polarization is likely to occur if the mean of the followers' ideological locations is a convex function of a direct consumer's location and/or the variance is a concave function. To provide an intuition for why convex mean leads to news polarization, suppose that the mean is increasing and convex over $[0,1 / 2] .^{2}$ This implies that the mean followers of two left-wing extremists are more closely located than those of two moderates. This can induce news polarization because, as the interval of direct consumers who share a news item expands, the marginal consumer's benefit from sharing it decreases more slowly when the news is located close to the extremists than when it is located close to the moderates. However, polarization does not entail choosing a news location equal to 0 or 1 because the media firm does not need further polarization of the news once it is extreme enough to induce the most extreme direct consumer to be indifferent between sharing the news and not, which we call the limit polarization.

In Section 4, we study competition between two media firms: firm $L$ and firm $R$. All direct consumers receive a news item from each firm, but each consumer can share at most one

\footnotetext{
${ }^{2}$ We consider a symmetric distribution of both direct readers and their followers around $1 / 2$.
} 
news item. We find that under mild conditions, as long as polarization occurs in the baseline model without competition, polarization also occurs under competition. When firm $R$ 's news becomes more polarized, additional right-wing consumers share its news whereas some moderate consumers stop sharing it. Compared to the same change in news location in the baseline model without competition, we find that the gain among right-wing consumers is the same whereas the loss among moderate consumers is typically smaller under competition. This is because the benefit that a moderate obtains by exercising the option of sharing firm $L$ 's news decreases as her position moves to the right whereas in the baseline model, consumers have no outside option, which is akin to having an outside option that does not depend on their location.

In Section 5, we provide three extensions of the baseline model. The first allows the firm to target an interval of consumers to show the news. When direct consumers are distributed uniformly, we find that it is a weakly dominant strategy for the firm to choose the same location of news as in the baseline model and to make the left end of the target interval coincide with the left marginal consumer who is indifferent between sharing the news and not. In the second extension, we add one additional layer of followers to consider resharing the shared news. We define a measure of homophily and show that news polarization in the baseline model implies news polarization in the extension as long as the homophily increases as consumers become more extreme. In the third extension, we study the depthmaximization strategy. We allow for many layers of followers and characterize the strategy that maximizes the depth of sharing, i.e., the number of times the news is shared following down the layers of communication. We find that as long as the attention tax is not small, depth maximization requires targeting the direct consumer with the lowest variance and locating the news at the mean location of the targeted consumer's followers.

Our work is closely related to the literature on demand-driven media bias (Gentzkow, Shapiro and Stone, 2015) that assumes that consumers enjoy reading news that confirms their biased beliefs. Mullainathan and Shleifer (2005) and Gabszewicz, Laussel and Sonnac (2001) use a Hotelling-type model to study media bias. Mullainathan and Shleifer (2005) consider price competition between two newspapers, and media bias emerges as each firm adopts a maximal differentiation strategy to soften price competition. By contrast, Gabszewicz, Laussel and Sonnac (2001) obtain minimal differentiation because the firms' main revenue sources are advertising and prices are constrained to be nonnegative, eliminating the force 
of differentiation to relax price competition. In addition, in both papers, a monopoly has no gain from choosing a location different from the middle. To the best of our knowledge, we are the first to embed social networks of followers into a model of demand-driven media bias to study how news sharing and news bias are shaped by the characteristics of the social networks. Empirical papers on demand-driven media bias include Gentzkow and Shapiro (2010) and Larcinese, Puglisi and Snyder (2011). Gentzkow and Shapiro (2010) find that readers have an economically significant preference for like-minded news and that firms respond strongly to consumer preferences. Larcinese, Puglisi and Snyder (2011) find evidence that newspapers cater to readers' partisan tastes on news about unemployment, trade deficits and budget deficits.

The literature on social media is mainly empirical and studies how social media affects voting, protests, xenophobia, polarization and the consumption of fake news (see Zhuravskaya, Petrova and Enikolopov, 2020, for a survey). Our paper is more related to the empirical papers studying polarization, which we reviewed at the beginning of the introduction. ${ }^{3}$ In particular, a very recent study by Levi (2021) based on a large field experiment on Facebook finds that newsfeed algorithm of Facebook limits exposure to counter-attitudinal news and thus increases polarization. There are several theoretical papers on social media. In terms of the categories of players, our paper is similar to Fainmesser and Galeotti (2020), which studies the interaction among marketers, influencers and followers as we study the interaction among media firms, direct consumers and followers; direct consumers in our model play the role of influencers in their model. Apart from this common point, the two papers are very different because they are not interested in news polarization and instead study competitive equilibria where each influencer chooses her amount of sponsored and organic content. Berman and Katona (2020) study how curation algorithm of a social media affects polarization when each receiver chooses the number of senders to follow and each sender chooses the quality of her content. Contrary to the empirical finding of Levi (2021), they find that curation algorithm can reduce polarization as it induces readers to follow a larger number of senders. Their research is complementary to ours as we plan to work on the role of newsfeed algorithm in our setting in the future. De Cornière and Sarvary (2018) study how content bundling by social media, i.e., social media shows news content together with

\footnotetext{
${ }^{3}$ See Tucker et al. (2018) for a review of the literature on social media, political polarization and political disinformation.
} 
user-generated content (UGC), affects the profit of newspapers and their incentive to invest in quality. However, they consider neither news sharing nor network structure of followers. Kranton and McAdams (2020) study how social networks affect news veracity when news producers should incur costs to produce true news. ${ }^{4}$ They find that veracity is low in dense networks when news producers are financed by advertising. Although we focus on a different question of news polarization, both papers share some common features. First, both study how social networks affect the supply side of news. Second, consumers who receive a news story directly from a producer decide to share or not the story with their followers. Last, each producer's revenue is proportional to the number of readers, ${ }^{5}$ which is determined by the number of consumers who share its news. Finally, what distinguishes our paper from all other papers is that we consider a rich structure of followers characterized by the mean and the variance of their ideological distributions and study how the distribution of the mean and the variance affects news polarization.

The next section presents our baseline model. All the proofs, but for those of Lemma 1 and Proposition 6 which are presented in the main text, are gathered in Appendix.

\section{The Baseline Model}

In this section, we present our baseline model, which has three categories of players: a media firm, direct consumers and followers.

The media firm and consumers The media firm chooses the ideological location of its news $y \in[0,1]$. We consider free news financed by advertising revenue. There is a continuum of consumers, who are also users of social media. Each consumer is located on the interval

\footnotetext{
${ }^{4}$ There are other papers studying news sharing in social networks. Campbell, Leister and Zenou (2019) build a dynamic model with two types of news (mass market and niche market) and two types of individuals. Each individual receives news from randomly sampled friends and shares one news item. They find that greater connectivity and homophily concurrently increase the prevalence of the niche market content and polarization. Bloch, Demange and Kranton (2018) study the transmission of rumors on social networks in a model with two possible states of nature and two types of agents (unbiased or biased). They find that a social network can serve as a filter: unbiased agents block messages from parts of the network that contain many biased agents.

${ }^{5}$ They also analyze a setting in which producers earn revenue from each consumer who takes a specific action based on their news story.
} 
$[0,1]$. The utility that a consumer located at $x$ obtains from reading a news located at $y$ is

$$
U(x, y)=u-(x-y)^{2},
$$

where $u>0$ and $(x-y)^{2}$ captures the disutility from the mismatch between the news opinion and the consumer's ideal opinion. Consumers read the news whenever they have access to it and obtain a nonnegative utility from reading it.

Direct consumers and followers We assume that the media firm can target an interval of consumers of length $l \in(0,1]$, where $l$ is a parameter that depends on the financial resources of the firm. Each targeted consumer receives news from the media firm. Those consumers who can be targeted by the media firm are called direct consumers. Direct consumers are distributed over $[0,1]$ according to the distribution function $F$ with density $f>0$, which is assumed to be symmetric around $1 / 2$. The particular case of $l=1$ is called no targeting.

Each direct consumer has followers, who are also called indirect consumers. Those who follow a direct consumer have access to the news only if it is shared by the direct consumer. We assume that every direct consumer has a distinct group of followers of equal measure, which is normalized to one. Given a direct consumer located at $x$, her followers are distributed according to density $\tilde{g}(\cdot ; x)$. We assume that the distribution of followers $z \in[0,1]$ of a consumer located at $x$ is symmetric to the distribution of followers of a consumer located at $1-x$ such that $\tilde{g}(z ; x)=\tilde{g}(1-z ; 1-x)$.

News sharing The existing literature from communication science and computer science identifies two motives for news sharing: an altruistic motive (Boyd, Golder and Lotan, 2010; Small, 2011; Holton et al., 2014) and a self-serving motive (Boyd, Golder and Lotan, 2010; Ma, Lee and Goh, 2011; Lee and Ma, 2012). We adopt the altruistic motive and assume that a direct consumer shares a news item if she reads it and if the average benefit that her followers obtain from reading it is larger than the attention tax $\tau$ she imposes on them by sharing the news. The attention tax represents the opportunity cost of attention, as news sharing by a direct consumer induces each follower to pay attention to it, which does not necessarily mean reading the whole news article. Using Simon (1971)'s expression, we assume that sharing news "consumes the attention of its recipients". A follower can discover the values of $u$ and $y$ by paying attention to the news and then decide whether to read it. 
Let $B(x, y)$ denote the average benefit that the followers of a direct consumer located at $x$ obtain when the latter shares news located at $y$. We have

$$
B(x, y)=\int_{0}^{1} \max \{U(z, y), 0\} \tilde{g}(z ; x) d z .
$$

Our altruistic approach regarding news sharing can be reconciled with standard utility maximization based on self-serving motives such as improving one's social status if the sharer attaches positive (negative) utilities to the positive (negative) reactions from her followers such as likes (dislikes) on Facebook or hearts on Twitter. ${ }^{6}$

Profit The media firm maximizes its advertising revenue, which is proportionate to the traffic to the firm's news site (Gentzkow, Shapiro and Stone, 2015). The traffic is equivalent to the measure of readers. Given the location of its news $y$, the profit of the firm is given by

$$
\pi(y)=(1-\alpha) D_{0}(y)+\alpha D_{1}(y)
$$

where $D_{0}(y)$ and $D_{1}(y)$ are the demand from the direct readers and that from the indirect readers, respectively, and $\alpha \in(0,1)$ captures the importance the firm assigns to the indirect demand relative to the direct one.

We assume that $u$ is large enough that every consumer has an incentive to read the news.

Assumption 1. $u$ is large enough that $U(x, y)>0$ for any $(x, y) \in[0,1]^{2}$.

This assumption greatly simplifies our analysis because under the assumption, every targeted consumer reads the news, and every follower also reads it as long as the news is shared. Therefore, profit maximization is equivalent to maximizing the measure of targeted consumers who share the news, which is called the breadth of news sharing:

\footnotetext{
${ }^{6}$ Suppose that a direct consumer receives a like (resp., dislike) from a follower located at $z$ whenever $U(z, y) \geq \tau$ (resp., $U(z, y)<\tau)$ and that the difference $U(z, y)-\tau$ (resp., $\tau-\max \{U(z, y), 0\})$ measures the intensity of the feedback received. Then, we have:

$$
\mathbb{E}(\text { intensity of likes })=\int_{\substack{U(z, y) \geq \tau \\ z \in[0,1]}}(U(z, y)-\tau) \tilde{g}(z ; x) d z,
$$

and

$$
\mathbb{E}(\text { intensity of dislikes })=\int_{\substack{U(z, y)<\tau \\ z \in[0,1]}}(\tau-\max \{U(z, y), 0\}) \tilde{g}(z ; x) d z .
$$

If a direct consumer shares news if and only if $\mathbb{E}$ (intensity of likes) $-\mathbb{E}$ (intensity of dislikes) $\geq 0$, then she shares news if and only if $B(x, y)-\tau \geq 0$.
} 
Definition 1 (Breadth of News Sharing). The breadth of sharing a news item is the measure of direct consumers who share it.

In the next section, we study the news location which maximizes the breadth of sharing. Due to the symmetry of the problem, if $y^{*}$ maximizes total demand, so does $1-y^{*}$. Therefore, it is sufficient to conduct analysis only over half of the interval. Henceforth, we restrict attention to $y \in[0,1 / 2]$ without loss of generality.

\section{Breadth-maximizing location: No targeting}

In this section, we study the breadth-maximizing location of news in the case of no targeting, which means that the target interval is equal to $[0,1]$. Targeting is considered in Section 5.1.

Let $\mu(x)$ and $\sigma^{2}(x)$ represent the mean and the variance, respectively, of the locations of the followers of a direct consumer located at $x$. Precisely,

$$
\mu(x)=\int_{0}^{1} z \tilde{g}(z ; x) d z \quad \text { and } \quad \sigma^{2}(x)=\int_{0}^{1}(z-\mu(x))^{2} \tilde{g}(z ; x) d z .
$$

We consider a general model in which the main primitives are the distribution of $\mu(x)$ and $\sigma^{2}(x)$ over $x \in[0,1]$. The next lemma shows that the benefit $B(x, y)$ is a function of $\mu(x)$ and $\sigma^{2}(x)$ regardless of the density of followers $\tilde{g}(z ; x)$. This is due to a quadratic loss in the utility from reading a news item.

Lemma 1. Under Assumption 1, the average benefit that the followers of a direct consumer located at $x$ obtain when the latter shares a news item located at $y$ is $B(x, y)=u-\sigma^{2}(x)-$ $(y-\mu(x))^{2}$.

Proof.

$$
\begin{aligned}
B(x, y) & =\mathbb{E}_{z}\left(u-(z-y)^{2}\right) \\
& =u-\mathbb{E}_{z}((z-\mu(x))-(y-\mu(x)))^{2} \\
& =u-\sigma^{2}(x)-(y-\mu(x))^{2}
\end{aligned}
$$

Therefore, given a direct consumer located at $x$, the benefit of news sharing decreases with the ideological dispersion of her followers $\sigma^{2}(x)$ and with the mismatch between the location of the news and the mean location of her followers $(y-\mu(x))^{2}$. 
We below introduce a technical assumption that adds regularity to $B(\cdot, y)$, thus simplifying our analysis. Under the assumption, the set of locations of direct consumers that share a news item is an interval, that is, if two direct consumers of distinct ideological locations are willing to share a news item, then any direct consumer located between the two is also willing to do so. We also assume that small changes in the ideological slant of a news item induce small changes in direct consumers' willingness to share it.

Assumption 2. For any $y, B(\cdot, y)$ is strictly quasiconcave and differentiable.

Let $x_{l}(y)$ and $x_{r}(y)$, with $x_{l}(y) \leq x_{r}(y)$, denote the limits of the interval of direct consumers who share a news item located at $y \in[0,1]$. From Assumption 1 , every follower reads the news if she has access to it. Thus, the indirect demand for a news item located at $y$ is equal to its breadth of sharing, that is,

$$
D_{1}(y)=\int_{x_{l}(y)}^{x_{r}(y)} f(x) d x=F\left(x_{r}(y)\right)-F\left(x_{l}(y)\right) .
$$

Let $y^{*}$ represent the breadth-maximizing news location. We first identify a straightforward case of no polarization.

Proposition 1. Under Assumptions 1 and 2, if the attention tax is low enough that even the most extreme consumer is willing to share news located at $1 / 2$, then no polarization $y^{*}=1 / 2$ maximizes the breadth of sharing because $D_{1}(1 / 2)=1$.

Therefore, henceforth, we consider $\tau>B(x=0, y=1 / 2)$. In this case, it is always possible to have a location $y$ (around $1 / 2$ ) such that both $x_{l}(y)>0$ and $x_{r}(y)<1$. The locations where either $x_{l}(y)=0$ or $x_{r}(y)=1$ are of special interest and will be called the limit polarization locations.

Definition 2 (Limit polarization locations). Let $\underline{y}$ (resp., $\bar{y}$ ) denote the closest location from the center such that $x_{l}(\underline{y})=0$ (resp., $\left.x_{r}(\bar{y})=1\right)$. We will refer to $y$ as the left limit-polarization location and to $\bar{y}$ as the right limit-polarization location.

From the symmetry of the distribution of followers, $\underline{y}=1-\bar{y}$.

The maximization of the indirect demand with respect to $y$ leads to the following firstorder condition

$$
\frac{\partial D_{1}}{\partial y}(y)=f\left(x_{r}\right) \frac{\partial x_{r}}{\partial y}-f\left(x_{l}\right) \frac{\partial x_{l}}{\partial y}
$$


Consider a consumer $\hat{x}(y) \in\left\{x_{l}(y), x_{r}(y)\right\}$ indifferent between sharing the news and not. Then, we have

$$
u-\sigma^{2}(\hat{x})-(y-\mu(\hat{x}))^{2}=\tau .
$$

Differentiating $\hat{x}$ with respect to $y$ in (2) yields

$$
\frac{\partial \hat{x}}{\partial y}=\frac{2(y-\mu)}{2 \mu_{x}(y-\mu)-\sigma_{x}^{2}} .
$$

We introduce our last assumption.

Assumption 3. We assume that there is some degree of homophily such that for any $y$, $\mu\left(x_{l}(y)\right)<y<\mu\left(x_{r}(y)\right)$.

Note first that the inequalities in the assumption require some homophily because without any homophily, $\mu(x)$ would be a constant that does not depend on $x$, clearly violating the inequalities. Suppose now that the assumption is not satisfied such that $\mu\left(x_{l}(y)\right)<$ $\mu\left(x_{r}(y)\right)<y$ holds (respectively, $y<\mu\left(x_{l}(y)\right)<\mu\left(x_{r}(y)\right)$ holds). Then, from Lemma 1 and Assumption 2, it is straightforward to see that moving $y$ to the left (respectively, to the right) increases the breadth. Therefore, the assumption makes breadth maximization less trivial.

The next two lemmas provide some intuitive results.

Lemma 2. Under Assumptions 1-3, $x_{l}$ is strictly increasing in $y \in[y, 1]$ and $x_{r}$ is strictly increasing in $y \in[0, \bar{y}]$.

From the Lemma above it follows that, under Assumptions 1-3, the breadth-maximizing news location $y^{*}$ always belongs to the interval $[\underline{y}, \bar{y}]$. To see that, consider first $y<\underline{y}$. Hence, $x_{l}(y)=0$ and $D_{1}(y)=F\left(x_{r}(y)\right)$. Then, $x_{r}$ strictly increasing in $[0, \underline{y}]$ implies that $y^{*} \geq \underline{y}$. Analogously, it is easy to see that $x_{l}$ strictly increasing in $[\bar{y}, 1]$ implies that $y^{*} \leq \bar{y}$.

Lemma 3. From the symmetry of the distribution of followers, we have that, for any $x$ and $y$,

1. $\mu(x)=1-\mu(1-x)$;

2. $\sigma^{2}(x)=\sigma^{2}(1-x)$;

3. $x_{r}(y)=1-x_{l}(1-y)$. 
We have the following result regarding (no) polarization of news:

Proposition 2. Assume that the density of direct readers is such that $f^{\prime}(x) \geq 0$ for $x \in$ $[0,1 / 2)$.

1. $x_{l}(y)$ being strictly convex for all $y \in[y, \bar{y}]$ is a sufficient condition for no polarization (i.e., $y^{*}=1 / 2$ ) to maximize the breadth of news sharing.

2. $x_{l}(y)$ being concave enough for all $y \in[y, \bar{y}]$ is a sufficient condition for the limit polarization strategy (i.e., $y^{*}=y$ or $y^{*}=\bar{y}$ ) to maximize the breadth of news sharing. If $F$ is uniformly distributed, $x_{l}(y)$ being strictly concave for all $y \in[\underline{y}, \bar{y}]$ is a sufficient condition for the limit polarization strategy to maximize the breadth.

Figure 1 graphically explains Proposition 2 when $F(x)$ is uniformly distributed.
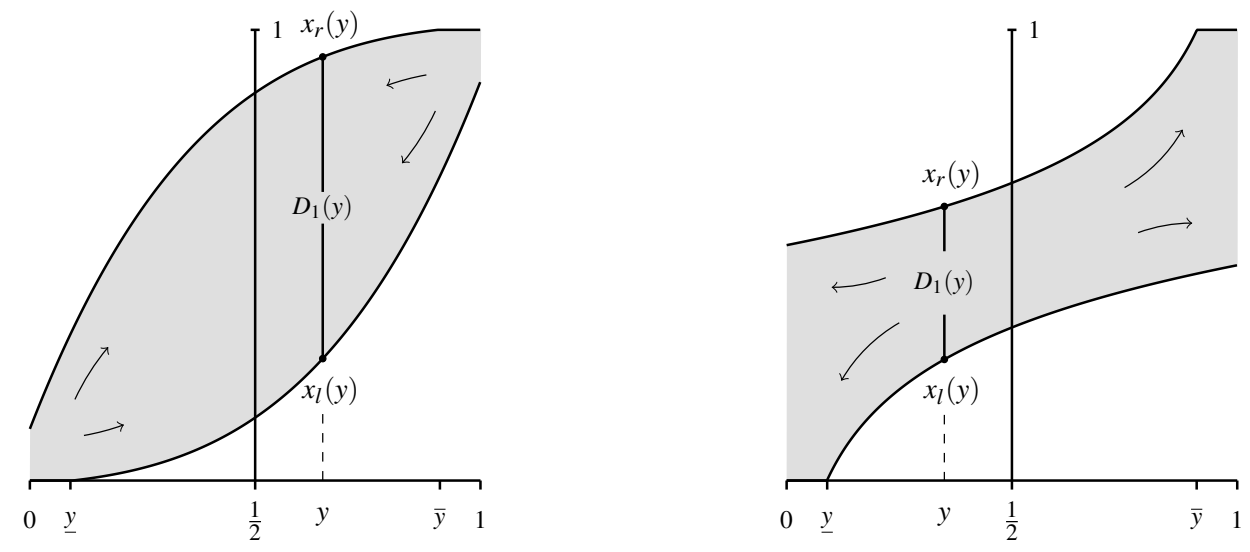

Figure 1: On the left, a convex $x_{l}(y)$ implies that indirect demand is maximized with no polarization, $y^{*}=1 / 2$. On the right, a concave $x_{l}(y)$ implies that indirect demand is maximized with limit polarization, $y^{*}=\underline{y}$ or $y^{*}=\bar{y}$.

Although the proposition is general, it is stated in terms of convexity or concavity of $x_{l}(y)$, the meaning of which is hard to grasp. Therefore, we consider the case of a uniform $F$ and try to understand the properties of $\mu(x)$ and $\sigma^{2}(x)$ that generate (no) polarization.

Suppose that $F$ is uniformly distributed. Consider $x_{l} \in(0,1 / 2)$. Then follows from equation (3)

$$
\frac{\partial^{2} x_{l}}{\partial y^{2}}=\frac{2\left(\sigma_{x}^{2}\right)^{2}-\left(2 \mu_{x x}(y-\mu)-\sigma_{x x}^{2}\right) 4(y-\mu)^{2}}{\left(2 \mu_{x}(y-\mu)-\sigma_{x}^{2}\right)^{3}} .
$$


Note that from equation (3), $x_{l}$ strictly increasing in $y$ together with Assumption 3 implies that $\left(2 \mu_{x}(y-\mu)-\sigma_{x}^{2}\right)$ is strictly positive. From this equation, we can analyze the following cases:

- Case 1: $\sigma^{2}$ constant (i.e., no variance effect).

$$
\frac{\partial^{2} x_{l}}{\partial y^{2}}=-\frac{\mu_{x x}}{\mu_{x}^{3}}
$$

Polarization occurs if $\mu$ is convex on $[0,1 / 2]$.

- Case 2: Both $\mu$ and $\sigma^{2}$ linear (i.e., no second derivative effect).

$$
\frac{\partial^{2} x_{l}}{\partial y^{2}}=\frac{2\left(\sigma_{x}^{2}\right)^{2}}{\left(2 \mu_{x}(y-\mu)-\sigma_{x}^{2}\right)^{3}}>0 .
$$

Polarization never occurs.

- Case 3: $\mu$ convex on $[0,1 / 2]$ and $\sigma^{2}$ linear.

$$
\frac{\partial^{2} x_{l}}{\partial y^{2}}=\frac{2\left(\sigma_{x}^{2}\right)^{2}-8 \mu_{x x}(y-\mu)^{3}}{\left(2 \mu_{x}(y-\mu)-\sigma_{x}^{2}\right)^{3}} .
$$

Polarization occurs if $\mu$ is convex enough on $[0,1 / 2]$.

- Case 4: $\mu$ linear and $\sigma^{2}$ concave on $[0,1 / 2]$.

$$
\frac{\partial^{2} x_{l}}{\partial y^{2}}=\frac{2\left(\sigma_{x}^{2}\right)^{2}+4 \sigma_{x x}^{2}(y-\mu)^{2}}{\left(2 \mu_{x}(y-\mu)-\sigma_{x}^{2}\right)^{3}} .
$$

Polarization occurs if $\sigma^{2}$ is concave enough on $[0,1 / 2]$.

The table provides the results for various cases.

Summarizing, we obtain our central result.

Proposition 3. Assume that $F(x)$ is uniformly distributed.

1. No polarization (i.e., $y^{*}=1 / 2$ ) occurs if (i) the mean $\mu(x)$ is concave enough or the variance $\sigma^{2}(x)$ is convex enough for $x \in[0,1 / 2]$, or (ii) both the mean $\mu(x)$ and variance $\sigma^{2}(x)$ are linear for $x \in[0,1 / 2]$.

2. The limit polarization (i.e., $y^{*}=y$ or $y^{*}=\bar{y}$ ) occurs if the mean $\mu(x)$ is convex enough or the variance $\sigma^{2}(x)$ is concave enough for $x \in[0,1 / 2]$. 


\begin{tabular}{lcccc} 
& Constant variance & Concave variance & Linear variance & Convex variance \\
\hline Concave mean & No & Yes, if variance con- & No & No \\
& & cave enough & \\
Linear mean & No & Yes, if variance con- & No & No \\
& cave enough & & \\
Convex mean & Yes & Yes, if mean convex Yes, if mean & Yes, if mean \\
& & enough or variance convex enough & convex enough \\
& concave enough & \\
\hline
\end{tabular}

Table 1: Cases where polarization occurs when $f$ is uniform.

We provide an intuition for why a convex mean $\mu(x)$ for $x \in[0,1 / 2]$ can lead to polarization. Note first that we expect $\mu(x)$ to increase with $x$. Then, a convex $\mu(x)$ implies that it increases in a increasing way for $x \in[0,1 / 2]$. This means that the mean followers of two left-wing extremists with some distance are more closely located than those of two moderates with the same distance (see Figure 2). Therefore, the benefit from news sharing $B(x, y)$ decreases more slowly when $x$ increases or decreases from $x^{\prime}$ satisfying $\mu\left(x^{\prime}\right)=y$ if the news is located close to the extremists than if it is located close to the moderates. This leads to the limit polarization.

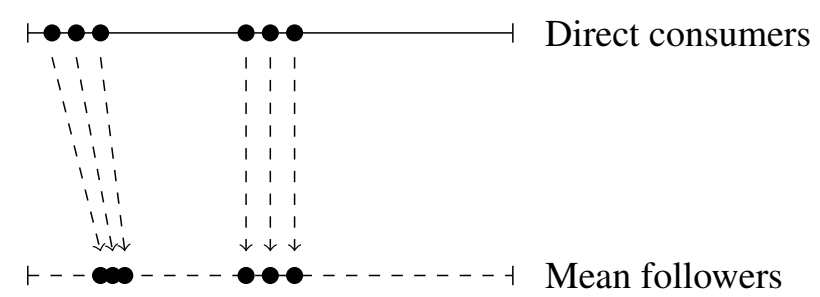

Figure 2: Convexity of $\mu(x)$ on $[0,1 / 2]$ means that the mean followers of extremists are more closely located to each other than those of moderates.

To provide an intuition for why a concave variance $\sigma^{2}(x)$ for $x \in[0,1 / 2]$ can lead to polarization, suppose that $\sigma^{2}(x)$ is increasing and concave for $x \in[0,1 / 2]$. Then, the symmetry around the middle implies that $\sigma^{2}(x)$ is concave for $x \in[0,1]$ and that it increases for $x \in[0,1 / 2]$ and decreases for $x \in[1 / 2,1]$. Now, consider $y<1 / 2$, and examine what happens when $y$ further moves to the left. This movement will induce some left-wing extremists to start to share the news while inducing some moderates to stop sharing it. However, when $\sigma^{2}(x)$ is concave, the decrease in $\sigma^{2}$ experienced by the former is larger than any change in 
$\sigma^{2}$ experienced by the latter, which leads to an overall expansion of the breadth. This logic implies that the limit polarization is optimal.

Let us now examine the effect of the distribution function of direct consumers. As long as the following function

$$
\phi(y)=f\left(x_{l}(y)\right) \frac{\partial x_{l}(y)}{\partial y}
$$

is increasing, it leads to $\frac{\partial D_{1}}{\partial y}>0$ for $y<1 / 2$ and $\frac{\partial D_{1}}{\partial y}<0$ for $y>1 / 2$, resulting in no polarization as the breadth-maximizing strategy. Analogously, $\phi$ decreasing leads to the limit polarization as the optimal strategy.

The derivative of $\phi$ is given by

$$
f^{\prime}\left(x_{l}\right)\left(\frac{\partial x_{l}}{\partial y}\right)^{2}+f\left(x_{l}\right) \frac{\partial^{2} x_{l}}{\partial y^{2}} .
$$

Previously, we fixed the sign of $f^{\prime}$ to be nonnegative on $[0,1 / 2)$. Now, note that $f^{\prime}(x) \geq 0$ (resp., $\leq 0)$ generates a force toward no polarization (resp., the limit polarization). U.S. experienced a dramatic increase in the polarization of partisan preferences over the past 40 years (Lazer et al., 2018). If it implies that $f$ is decreasing over $[0,1 / 2)$, then this increase in the polarization of preferences makes news polarization more likely in our model.

\section{Competition}

We here consider competition between two media firms, $L$ and $R$. Each firm $i$ (with $i=L, R$ ) chooses the ideological position of its news $y_{i}$. Without loss of generality, we can consider $y_{L} \leq y_{R}$ in equilibrium. We consider no targeting and assume that all direct consumers receive both news items, but each of them shares at most one news item. A direct consumer shares the news that generates the larger average benefit conditional on it being larger than the attention tax.

Let $\left[x_{l}\left(y_{L}\right), x_{r}\left(y_{L}\right)\right]$ (resp. $\left.\left[x_{l}\left(y_{R}\right), x_{r}\left(y_{R}\right)\right]\right)$ be the interval of locations of direct consumers who prefer sharing the news from firm $L$ (resp. firm $R$ ) over not sharing any news. The functions $x_{l}(\cdot)$ and $x_{r}(\cdot)$ are determined in the same way as in the baseline model. Suppose that $x_{l}\left(y_{R}\right)<x_{r}\left(y_{L}\right)$. A direct consumer is indifferent between sharing $L$ 's news and sharing $R$ 's news if she is located at $x_{m}=x_{m}\left(y_{L}, y_{R}\right)$ such that $B\left(x_{m}, y_{L}\right)=B\left(x_{m}, y_{R}\right)$, which is equivalent to $\mu\left(x_{m}\right)=\left(y_{L}+y_{R}\right) / 2$. Therefore, the firms' respective indirect demands are

$$
D_{L}\left(y_{L}, y_{R}\right)=F\left(x_{m}\left(y_{L}, y_{R}\right)\right)-F\left(x_{l}\left(y_{L}\right)\right)
$$


and

$$
D_{R}\left(y_{L}, y_{R}\right)=F\left(x_{r}\left(y_{R}\right)\right)-F\left(x_{m}\left(y_{L}, y_{R}\right)\right) .
$$

Let $\left(y_{L}^{*}, y_{R}^{*}\right)$ represent the equilibrium news locations under competition between the two media firms. The next lemma shows an intuitive result:

Lemma 4. Under competition between the two media firms, if an equilibrium exists, we must have $y_{L}^{*} \leq 1 / 2 \leq y_{R}^{*}$.

If the attention tax is low enough, we have $x_{l}(1 / 2)=0$ and $x_{r}(1 / 2)=1$. In that case, as in the baseline model, we have no polarization.

Proposition 4. Under competition between the two media firms, if the attention tax is low enough that even the most extreme consumer is willing to share ideologically neutral news, there is a unique equilibrium in which both firms adopt the no-polarization strategy, i.e., $y_{L}^{*}=y_{R}^{*}=1 / 2$.

The above proposition is similar to the result obtained by Gabszewicz, Laussel and Sonnac (2001) in a Hotelling model in which two newspapers compete in prices. When the nonnegative pricing constraint of newspapers binds due to high advertising revenue, they find that both newspapers locate at the middle. However, they consider neither social media nor news sharing.

We now consider the case of $\tau>B(x=0, y=1 / 2)$ and study when it is optimal for each firm to adopt the limit polarization. Suppose that $F$ is uniform. The next proposition shows that under mild conditions, the limit polarization in the baseline model without competition implies the limit polarization under competition between the two media firms.

Proposition 5. Suppose that $F$ is uniformly distributed. The limit polarization in the baseline model without competition implies the limit polarization under competition between the two media firms $y_{L}^{*}=\underline{y}$ and $y_{R}^{*}=\bar{y}$ whenever, for $x \in[0,1 / 2]$,

1. $\mu(x)$ is convex and $\sigma_{x}^{2} \leq 0$ or $\sigma_{x}^{2}>0$ but small; or

2. $\mu(x)$ is linear and $\sigma^{2}(x)$ is concave but with a slope not very negative.

Note first that the conditions in the proposition such as convex mean and concave variance are consistent with the limit polarization in the baseline model without competition (see 
Proposition 3). To provide an intuition for the result, suppose that firm $L$ chooses some $y_{L}<\bar{y}$ given $y_{R}=\bar{y}$. Then, the interval of direct consumers who share $L$ 's news is given by $\left[x_{l}\left(y_{L}\right), x_{m}\left(y_{L}, \bar{y}\right)\right]$. Compare this interval with the interval of sharers $\left[x_{l}\left(y_{L}\right), x_{r}\left(y_{L}\right)\right]$ for a monopolist choosing the same $y=y_{L}$. Consider now moving $y_{L}$ slightly to the left. Then, the induced decrease in $x_{l}\left(y_{L}\right)$ is the same in both intervals. However, the induced decrease in $x_{m}\left(y_{L}, \bar{y}\right)$ is typically smaller than the one in $x_{r}\left(y_{L}\right)$. This is because the marginal consumer under competition, who is located at $x_{m}\left(y_{L}, \bar{y}\right)$, has an outside option of sharing news from $R$ of which the benefit increases with her location, whereas the marginal consumer in the baseline model without competition, who is located at $x_{r}\left(y_{L}\right)$, has no outside option, which is akin to having an outside option that does not depend on her location. As $y_{L}$ moves to the left, the location of the marginal consumer $x_{m}\left(y_{L}, \bar{y}\right)$ also moves to the left, which in turn makes less attractive the outside option of sharing news from $R$ (see Figure 3 ).

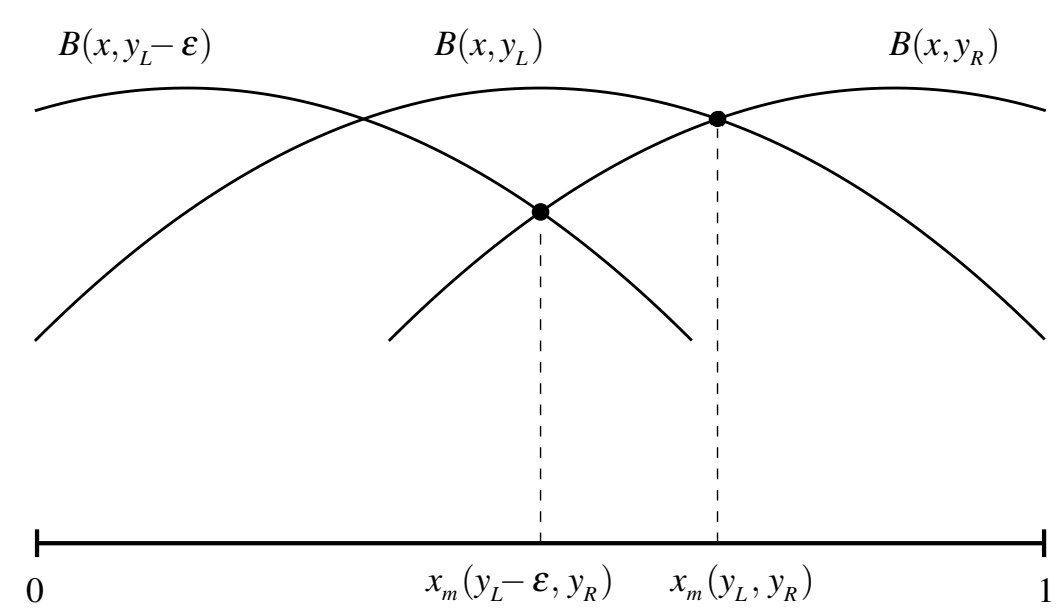

Figure 3: In the figure, firm $L$ moves the location of its news to the left given the location of $R$ 's news.

\section{Extensions}

In this section, we provide three extensions of the baseline model. 


\section{$5.1 \quad$ Targeting}

Here, we extend the baseline model by enabling the media firm to target an interval of direct readers. For instance, the Macedonian teenagers, mentioned in the introduction, purchased bogus Facebook accounts and used them to target certain profiles of users to spread their fake news.

Suppose that the media firm can target direct consumers belonging to an interval of given length $l \in[0,1)$ to send its news. Suppose also that direct consumers are uniformly distributed. Hence, the media firm now should choose not only the location of its news $y$ but also the target interval $[a, a+l] \subset[0,1]$ by choosing $a$. We show that it is a weakly dominant strategy to choose the optimal location without targeting (i.e., $\left.y=y^{*}\right)$ and $a=x_{l}\left(y^{*}\right)$.

Proposition 6 (Targeting Strategy). Suppose that the media firm can choose both the news location $y$ and the target interval of direct consumers $[a, a+l] \subset[0,1]$ by choosing a where $l \in[0,1)$ is exogenously given. Suppose that $F$ is uniformly distributed. It is a weakly dominant strategy for the firm to choose $y=y^{*}$ and $a=x_{l}\left(y^{*}\right)$.

Proof. We need to distinguish two cases: either $a+l \leq x_{r}\left(y^{*}\right)$ or $a+l>x_{r}\left(y^{*}\right)$. In the first case, all targeted consumers share the news and one cannot do better. In the second case, all consumers in the interval $\left[x_{l}\left(y^{*}\right), x_{r}\left(y^{*}\right)\right]$ are targeted and share the news and hence one cannot do better either.

\section{$5.2 \quad$ Resharing}

Here, we extend the baseline model by adding one additional layer of communication: the indirect consumers can share news with their own followers. The utility from reading news is given by $U(x, y)=u-(x-y)^{2}$ as before. We maintain the assumption that each group of followers is distinct at any layer.

The profit of the firm is now given by

$$
\pi(y)=\sum_{n=0}^{2} \alpha_{n} D_{n}(y)
$$

where $D_{n}(y)$ denotes the demand from consumers at the $n$-th layer of communication and $\alpha_{n} \geq 0$ is a weight for the demand from consumers at the $n$-th layer.

We below introduce a measure of the degree of homophily. 
Definition 3 (Homophily). Given an ideological segment $[a, a+l]$, let $h_{2}(a, l)$ denote the measure of 2 nd layer consumers whose ideological locations belong to the interval $[a, a+l]$ and follow a first-layer consumer located in the same interval. We refer to $h_{2}(a, l)$ as the 2 d layer homophily on the interval $[a, a+l]$. Additionally, we say that the homophily is extremal (central) if the homophily increases toward the extremes (resp., center), that is,

$$
\frac{\partial h_{2}}{\partial a}(a, l)= \begin{cases}<0(\text { resp. },>0) & \text { for } a \in[0,1 / 2-l / 2) \\ >0(\text { resp. }, \quad<0) & \text { for } a \in(1 / 2-l / 2,1-l] .\end{cases}
$$

We have the following result:

Proposition 7 (Resharing). Suppose that $F$ is uniformly distributed.

1. No polarization in the baseline model implies no polarization in the extension with one additional layer if homophily is central.

2. The limit polarization in the baseline model implies the limit polarization in the extension with one additional layer if homophily is extremal.

Asymmetries in the degree of homophily are observed on Twitter by Boutyline and Willer (2017). Using a data set of the entire Twitter network from 2009, the authors find that more extreme individuals tend to be more homophilous than more moderate ones. Then, homophily is extremal and, according to the above proposition, the limit polarization in the baseline model implies the limit polarization in the extension.

\subsection{The depth-maximization strategy}

Here, we provide an extension of the baseline model that focuses on the depth of news sharing. For this purpose, we allow for the resharing of news following down many layers of communication. We maintain the assumption that each group of followers is distinct at any layer.

For the resharing to stop at some layer, we assume that the constant $u$ depreciates when the layer of sharing increases. That is, the utility that a consumer located at $x$ obtains from reading a news item located at $y$ after it has been shared $n=1,2, \ldots$ times is

$$
U_{n}(x, y)=\delta^{n-1} u-(x-y)^{2}, \quad \text { with } \delta \in(0,1)
$$


and the benefit generated by sharing the news item one additional time is given by

$$
B_{n+1}(x, y)=\int_{0}^{1} \max \left\{U_{n+1}(z, y), 0\right\} \tilde{g}(z ; x) d z .
$$

For the sake of computational simplicity, we adopt a continuous version of the problem. Let $t \in[0,+\infty)$ be the time at which a news item located at $y$ reaches an indirect consumer located at $x$. Then, a consumer's utility from reading the news and her benefit of resharing it will be given, respectively, by

$$
U_{t}(x, y)=\delta^{t} u-(x-y)^{2} \quad \text { and } \quad B_{t}(x, y)=\int_{0}^{1} \max \left\{U_{t}(z, y), 0\right\} \tilde{g}(z ; x) d z .
$$

Definition 4 (Depth of News Sharing). We define the depth of news sharing as the maximum number of times that a news item is shared following down the hierarchical layers of communication.

We study the depth-maximization strategy and allow the firm to target only one direct consumer without loss of generality. Hence, the firm chooses the location of the targeted consumer and the location of the news item. We have the following result:

Proposition 8 (Depth-Maximizing Strategy). Suppose that the attention tax is not small. The media firm's optimal strategy to maximize the depth of news sharing is characterized as follows:

1. It targets the consumer located at $x_{\text {depth }}^{*}$ whose $\sigma^{2}(x)$ is equal to $\min _{x \in[0,1 / 2]} \sigma^{2}(x)$.

2. It chooses the location of the news item $y_{\text {depth }}^{*}=\mu\left(x_{\text {depth }}^{*}\right)$.

Corollary 1. Suppose that the attention tax is not small.

1. Depth maximization leads to some polarization as long as $\sigma^{2}(1 / 2)>\min _{x \in[0,1 / 2]} \sigma^{2}(x)$;

2. If $\sigma^{2}(x)$ is increasing on $[0,1 / 2]$, it leads to $x_{\text {depth }}^{*}=0$.

The assumption that the attention tax is not small means that for consumers at the target location, as the news depreciates, the net benefit of sharing it becomes zero before the utility of reading it becomes zero. For a given consumer, her benefit from sharing a news item is maximized when the item has the same location as that of her mean follower. From 
equation (1), this leads to a benefit equal to $u-\sigma^{2}(x)$. Therefore, it is optimal to target the location of the direct consumer with the lowest variance $\sigma^{2}(x)$.

If one considers that false political news tends to be hyperpartisan (Silverman et al., 2016), our results provide an explanation for the findings of Vosoughi, Roy and Aral (2018) that false political news diffused deeper and more broadly than true news. The authors suggest that the degree of novelty and the extent to which the news is emotionally charged may explain their findings. Our results suggest that their findings may also be explained by the structure of social networks: a news item appealing to a group of consumers who have very homogeneous ideological preferences tend to diffuse deeply.

\section{Conclusion}

We have studied how the distribution of followers' ideological preferences shapes a user's incentive to share news on social media and how this, in turn, does or not create incentives for a media firm to provide partisan content. In particular, we have focused on how the distribution of the mean and the variance of followers' ideological locations affect the ideological location of news. We have found that both a convex mean and a concave variance contribute to polarization when a media firm maximizes the breadth of news sharing.

Although we assumed that both the distribution of direct consumers and that of followers are symmetric around $1 / 2$, in reality, more conservative individuals are more homophilous than more liberal ones (Boutyline and Willer (2017)). It would be interesting to extend our framework to incorporate such asymmetry.

We believe that the most interesting avenue for future research is to examine the role of news feed algorithms (Berman and Katona, 2020). In the case of Facebook, after a user decides to share news with her friends, the algorithm determines which subset of her friends will be exposed to the news. If a social media platform can employ an algorithm to maximize the amount of time users spend on the platform, will such an engagementmaximizing algorithm lead to more or less polarization of news?

Another interesting revenue for future research consists in incorporating consumers' actions such as voting and study how news consumption and polarization influences their actions (Galeotti and Mattozzi, 2011, Kranton and McAdams, 2020). 


\section{References}

Allcott, Hunt, Luca Braghieri, Sarah Eichmeyer, and Matthew Gentzkow. 2020. "The Welfare Effects of Social Media." American Economic Review, 110(3): 629-76.

Bakshy, Eytan, Solomon Messing, and Lada Adamic. 2015. "Exposure to ideologically diverse news and opinion on Facebook." Science.

Barberá, Pablo. 2015. "How Social Media Reduces Mass Political Polarization. Evidence from Germany, Spain, and the U.S." Working paper.

Berman, Ron, and Zsolt Katona. 2020. "Curation Algorithms and Filter Bubbles in Social Networks." Marketing Science, 39(2): 296-316.

Bloch, Francis, Gabrielle Demange, and Rachel Kranton. 2018. "Rumors and Social Networks." International Economic Review, 59(2): 421-448.

Boutyline, Andrei, and Robb Willer. 2017. "The Social Structure of Political Echo Chambers: Variation in Ideological Homophily in Online Networks." Political Psychology, 38(3): 551-569.

Boxell, Levi, Matthew Gentzkow, and Jesse M. Shapiro. 2017. "Greater Internet use is not associated with faster growth in political polarization among US demographic groups." Proceedings of the National Academy of Sciences, 114(40): 10612-10617.

Boyd, Danah, Scott Golder, and Gilad Lotan. 2010. "Tweet, Tweet, Retweet: Conversational Aspects of Retweeting on Twitter." 1-10.

Campbell, Arthur, C. Matthew Leister, and Yves Zenou. 2019. "Scial Media and Polarization." Working paper.

De Cornière, Alexandre, and Miklos Sarvary. 2018. "Social Media and News: Attention Capture via Content Bundling." Working paper.

Fainmesser, Itay P., and Andrea Galeotti. 2020. "The Market for Online Influence." Forthcoming.

Gabszewicz, Jean J., Dider Laussel, and Nathalie Sonnac. 2001. "Press advertising and the ascent of the 'Pensée Unique'." European Economic Review, 45(4): 641-651. 
Galeotti, Andrea, and Andrea Mattozzi. 2011. "Personal Influence": Social Context and Political Competition." American Economic Journal: Microeconomics, 3(1): 307-327.

Gentzkow, Matthew, and Jesse M. Shapiro. 2010. "What Drives Media Slant? Evidence From U.S. Daily Newspapers." Econometrica, 78(1): 35-71.

Gentzkow, Matthew, Jesse M. Shapiro, and Daniel F. Stone. 2015. "Chapter 14 - Media Bias in the Marketplace: Theory." In Handbook of Media Economics. Vol. 1 of Handbook of Media Economics, , ed. Simon P. Anderson, Joel Waldfogel and David Strömberg, 623-645. North-Holland.

Halberstam, Yosh, and Brian Knight. 2016. "Homophily, group size, and the diffusion of political information in social networks: Evidence from Twitter." Journal of Public Economics, 143: 73-88.

Holton, Avery E., Kang Baek, Mark Coddington, and Carolyn Yaschur. 2014. "Seeking and Sharing: Motivations for Linking on Twitter." Communication Research Reports, 31(1): 33-40.

Kranton, Rachel, and David McAdams. 2020. "Social Networks and the Market for News." Working Paper.

Kümpel, Anna Sophie, Veronika Karnowski, and Till Keyling. 2015. "News Sharing in Social Media: A Review of Current Research on News Sharing Users, Content, and Networks." Social Media + Society, 1(2).

Larcinese, Valentino, Riccardo Puglisi, and James M. Snyder. 2011. "Partisan bias in economic news: Evidence on the agenda-setting behavior of U.S. newspapers." Journal of Public Economics, 95(9): 1178-1189.

Lazer, David M. J., Matthew A. Baum, Yochai Benkler, Adam J. Berinsky, Kelly M. Greenhill, Filippo Menczer, Miriam J. Metzger, Brendan Nyhan, Gordon Pennycook, David Rothschild, Michael Schudson, Steven A. Sloman, Cass R. Sunstein, Emily A. Thorson, Duncan J. Watts, and Jonathan L. Zittrain. 2018. "The science of fake news." Science, 359(6380): 1094-1096. 
Lee, Chei Sian, and Long Ma. 2012. "News sharing in social media: The effect of gratifications and prior experience." Computers in Human Behavior, 28(2): 331-339.

Levi, Ro'ee. 2021. "Social Media, News Consumption, and Polarization: Evidence from a Field Experiment." American Economic Review, 111(3): 831-70.

Madio, Leonardo, and Martin Quinn. 2020. "User-generated Content, Strategic Moderation, and Advertising." Working paper.

Ma, Long, Chei Sian Lee, and Dion Hoe-Lian Goh. 2011. "That's News to Me: The Influence of Perceived Gratifications and Personal Experience on News Sharing in Social Media." 141-144. New York, NY, USA:ACM.

Matsa, Katerina Eva, and Elisa Shearer. 2018. "News Use Across Social Media Platforms 2018." Pew Research Center.

Mullainathan, Sendhil, and Andrei Shleifer. 2005. "The Market for News." American Economic Review, 95(4): 1031-1053.

NPR. 2016. "Fake News Expert On How False Stories Spread And Why People Believe Them." December 14. https://www.npr.org/2016/12/14/505547295/fake-news-expert-onhow-false-stories-spread-and-why-people-believe-them.

Pariser, Eli. 2011. The Filter Bubble: What the Internet is Hiding from You. Penguin.

Silverman, Craig, and Lawrence Alexander. 2016. "How Teens In The Balkans Are Duping Trump Supporters With Fake News." BuzzFeed.

Silverman, Craig, Lauren Strapagiel, Hamza Shaban, Ellie Hall, and Jeremy Singer-Vine. 2016. "Hyperpartisan Facebook Pages Are Publishing False And Misleading Information At An Alarming Rate." BuzzFeed.

Simon, Herbert A. 1971. "Designing Organizations for an Information-Rich World." Computers, Communications, and the Public Interest, , ed. Martin Greenberger, 40-41. Baltimore, MD:Johns Hopkins Press.

Small, Tamara A. 2011. "What the hashtag?" Information, Communication \& Society, 14(6): 872-895. 
Sunstein, Cass R. 2017. \#Republic: Divided Democracy in the Age of Social Media. Princeton University Press.

Tucker, Joshua Aaron, Andrew Guess, Pablo Barberá, Cristian Vaccari, Alexandra Siegel, Sergey Sanovich, Denis Stukal, and Brendan Nyhan. 2018. "Social Media, Political Polarization, and Political Disinformation: A Review of the Scientific Literature." William and Flora Hewlett Foundation.

Vosoughi, Soroush, Deb Roy, and Sinan Aral. 2018. "The spread of true and false news online." Science, 359(6380): 1146-1151.

Yanagizawa-Drott, David, Maria Petrova, and Ruben Enikolopov. 2019. "Echo Chambers: Does Online Network Structure Affect Political Polarization?" Working Paper.

Zhuravskaya, Ekaterina, Maria Petrova, and Ruben Enikolopov. 2020. "Political Effects of the Internet and Social Media." Annual Review of Economics, 12(1): 415-38.

\section{Appendix}

\section{Proof of Proposition 1}

The quasiconcavity of the benefit function implies that, for any $y, B(x, y) \geq \min \{B(0, y), B(1, y)\}$, for all $x$. Additionally, from the symmetry of the problem, $\max _{y \in[0,1]} \min \{B(0, y), B(1, y)\}=$ $B(0,1 / 2)(=B(1,1 / 2))$.

Therefore, if $\tau \leq B(0,1 / 2)$, by locating its news at $y^{*}=1 / 2$ the media firm can induce sharing from all direct consumers, thus maximizing the breadth of news sharing.

\section{Proof of Lemma 2}

Note that

$$
\frac{\partial B(x, y)}{\partial x}=2 \mu_{x}(y-\mu)-\sigma_{x}^{2}
$$

In addition, from $B(\cdot, y)$ being quasiconcave and differentiable follows that

$$
\left.\frac{\partial B(x, y)}{\partial x}\right|_{x=x_{l}(y)}>0 \quad \text { and }\left.\quad \frac{\partial B(x, y)}{\partial x}\right|_{x=x_{r}(y)}<0
$$


Taking this together with Assumption 3 and equation (3) implies that both $\partial x_{l} / \partial y$ and $\partial x_{r} / \partial y$ are positive whenever $x_{l}$ and $x_{r}$ satisfy equation (2).

\section{Proof of Lemma 3}

1 and 2 follow directly from our assumption that $\tilde{g}(z ; x)=\tilde{g}(1-z ; 1-x)$.

To prove 3 , we start by noting that 1 and 2 imply that $B(x, y)=B(1-x, 1-y)$ for all $x, y \in[0,1]$. From that, if $x_{l}(y)$ and $x_{r}(y)$ are solutions for $B(\hat{x}, y)=\tau$, then $1-x_{l}(y)$ and $1-x_{r}(y)$ are solutions for $B(\hat{x}, 1-y)=\tau$. Finally, as $x_{l}(y)<x_{r}(y)$ implies $1-x_{l}(y)>1-x_{r}(y)$, we have that $x_{l}(1-y)=1-x_{r}(y)$.

\section{Proof of Proposition 2}

1. Take $y<1 / 2$.

(a) From Lemma 2 we have that $x_{l}$ is strictly increasing. Then, $x_{l}(y)<x_{l}(1-y)$ for any $y<1 / 2$. Additionally, we have that $x_{l}(y)<x_{r}(y)=1-x_{l}(1-y)$, i.e., the distance between $x_{l}(y)$ and 0 is shorter than the distance between $x_{l}(1-y)$ and 1 . Hence, $f^{\prime} \geq 0$ for $x \in[0,1 / 2)$ and symmetry of $f$ imply that $f\left(x_{l}(y)\right) \leq$ $f\left(x_{l}(1-y)\right)=f\left(x_{r}(y)\right) ;$

(b) $x_{l}$ strictly convex implies $\frac{\partial x_{l}(y)}{\partial y}<\frac{\partial x_{l}(1-y)}{\partial y}$; and

(c) From Lemma 3 we have $x_{r}(y)=1-x_{l}(1-y)$. Hence, $\frac{\partial x_{r}(y)}{\partial y}=\frac{\partial x_{l}(1-y)}{\partial y}$.

From (a)-(c) and from the symmetry of $f$ follows that

$$
\frac{\partial D_{1}}{\partial y}(y)=f\left(x_{r}(y)\right) \frac{\partial x_{r}}{\partial y}(y)-f\left(x_{l}(y)\right) \frac{\partial x_{l}}{\partial y}(y)>0 .
$$

It is straightforward to verify that $y>1 / 2$ implies $\frac{\partial D_{1}}{\partial y}(y)<0$. Therefore, the breadth is maximized at $y^{*}=1 / 2$.

2. For any $y<\underline{y}$, we have that $x_{l}(y)=0$ and

$$
\frac{\partial D_{1}}{\partial y}(y)=f\left(x_{r}(y)\right) \frac{\partial x_{r}}{\partial y}(y) \geq 0
$$


On the other hand, for any $y<y<1 / 2$, we have that if $x_{l}(y)$ is concave enough such that

$$
\frac{\partial^{2} x_{l}}{\partial y^{2}}<-\frac{f^{\prime}\left(x_{l}\right)}{f\left(x_{l}\right)}\left(\frac{\partial x_{l}}{\partial y}\right)^{2}
$$

then the product $f\left(x_{l}\right) \frac{\partial x_{l}}{\partial y}$ is decreasing in $y$, and we have that

$$
\frac{\partial D_{1}}{\partial y}(y)=f\left(x_{l}(1-y)\right) \frac{\partial x_{l}}{\partial y}(1-y)-f\left(x_{l}(y)\right) \frac{\partial x_{l}}{\partial y}(y)<0 .
$$

Hence, the limit polarization strategy is optimal.

\section{Proof of Proposition 3}

When direct readers are uniformly distributed, it follows that

$$
\begin{aligned}
\frac{\partial D_{1}}{\partial y}(y) & =\frac{\partial x_{r}}{\partial y}(y)-\frac{\partial x_{l}}{\partial y}(y) \\
& =\frac{\partial x_{l}}{\partial y}(1-y)-\frac{\partial x_{l}}{\partial y}(y) .
\end{aligned}
$$

Therefore, no polarization (respectively, limit polarization) occurs if $x_{l}(y)$ is convex (respectively, concave) on $[y, \bar{y}]$. From equation (3) we have that

$$
\frac{\partial^{2} x_{l}}{\partial y^{2}}=\frac{2\left(\sigma_{x}^{2}\left(x_{l}\right)\right)^{2}-\left(2 \mu_{x x}\left(x_{l}\right)\left(y-\mu\left(x_{l}\right)\right)-\sigma_{x x}^{2}\left(x_{l}\right)\right) 4\left(y-\mu\left(x_{l}\right)\right)^{2}}{\left(2 \mu_{x}\left(x_{l}\right)\left(y-\mu\left(x_{l}\right)\right)-\sigma_{x}^{2}\left(x_{l}\right)\right)^{3}} .
$$

Hence,

$$
\frac{\partial^{2} x_{l}}{\partial y^{2}} \gtrless 0 \quad \text { if and only if } \quad 2 \mu_{x x}\left(x_{l}\right)\left(y-\mu\left(x_{l}\right)\right)-\sigma_{x x}^{2}\left(x_{l}\right) \lessgtr \frac{1}{2}\left(\frac{\sigma_{x}^{2}\left(x_{l}\right)}{y-\mu\left(x_{l}\right)}\right)^{2},
$$

and the proposition follows.

\section{Proof of Lemma 4}

Let $\left(y_{L}^{*}, y_{R}^{*}\right)$ be an equilibrium with $y_{L}^{*} \leq y_{R}^{*}$.

We prove the result by contradiction. Suppose, for example, that $y_{L}^{*} \leq y_{R}^{*}<1 / 2$. Then, $D_{L}\left(y_{L}^{*}, y_{R}^{*}\right)=x_{m}\left(y_{L}^{*}, y_{R}^{*}\right)-x_{l}\left(y_{L}^{*}\right)$ and

$$
\begin{aligned}
D_{L}\left(1-y_{L}^{*}, y_{R}^{*}\right) & =x_{r}\left(1-y_{L}^{*}\right)-x_{m}\left(1-y_{L}^{*}, y_{R}^{*}\right) \\
& =\left[1-x_{l}\left(y_{L}^{*}\right)\right]-x_{m}\left(1-y_{L}^{*}, y_{R}^{*}\right) \\
& =\left[1-x_{m}\left(1-y_{L}^{*}, y_{R}^{*}\right)\right]-x_{l}\left(y_{L}^{*}\right) .
\end{aligned}
$$


As $\left(y_{L}^{*}, y_{R}^{*}\right)$ is an equilibrium, we must have $D_{L}\left(y_{L}^{*}, y_{R}^{*}\right) \geq D_{L}\left(1-y_{L}^{*}, y_{R}^{*}\right)$ or, equivalently, $x_{m}\left(y_{L}^{*}, y_{R}^{*}\right) \geq 1-x_{m}\left(1-y_{L}^{*}, y_{R}^{*}\right)$.

As the mean function $\mu$ is increasing and that we have

$$
\mu\left(x_{m}\left(y_{L}^{*}, y_{R}^{*}\right)\right)=\frac{y_{L}^{*}+y_{R}^{*}}{2}
$$

and

$$
\begin{aligned}
\mu\left(1-x_{m}\left(1-y_{L}^{*}, y_{R}^{*}\right)\right) & =1-\mu\left(x_{m}\left(1-y_{L}^{*}, y_{R}^{*}\right)\right) \\
& =1-\frac{1-y_{L}^{*}+y_{R}^{*}}{2}=\frac{y_{L}^{*}+\left(1-y_{R}^{*}\right)}{2},
\end{aligned}
$$

$x_{m}\left(y_{L}^{*}, y_{R}^{*}\right) \geq 1-x_{m}\left(1-y_{L}^{*}, y_{R}^{*}\right)$ if and only if $y_{R}^{*} \geq 1-y_{R}^{*}$, that is, $y_{R}^{*} \geq 1 / 2$, which contradicts our initial hypothesis that $y_{R}^{*}<1 / 2$.

\section{Proof of Proposition 4}

Clearly, $y_{L}^{*}=y_{R}^{*}=1 / 2$ is an equilibrium since by deviating from the center a firm does not increases the number of sharing from extremists (since all of them were already sharing) while it loses sharing from some moderates who now prefer to share the news of its competitor.

In any other tentative equilibrium where one firm, say firm $L$, plays $y_{L} \neq 1 / 2$, the best reply for firm $R$ is to chose a location $y_{R}$ between $y_{L}$ and $1 / 2$, the closest possible of $y_{L}$. But since whenever $y_{R} \neq 1 / 2$ then the best reply of firm $L$ is to choose location $y_{L}$ between $y_{R}$ and $1 / 2$, the closest possible of $y_{R}$, the only possible equilibrium is $y_{L}^{*}=y_{R}^{*}=1 / 2$.

\section{Proof of Proposition 5}

Limit polarization occurs in the baseline model without competition when we have

$$
\frac{\partial D_{1}}{\partial y}(y)=\frac{\partial x_{r}}{\partial y}(y)-\frac{\partial x_{l}}{\partial y}(y)= \begin{cases}<0 & \text { for } y \in(\underline{y}, 1 / 2) \\ >0 & \text { for } y \in(1 / 2, \bar{y})\end{cases}
$$

Under competition between the two media firms, we consider the deviation of the $L$ firm given that the $R$ firm chooses $y_{R}=\bar{y}$. Then we have

$$
\frac{\partial D_{L}}{\partial y_{L}}(y, \bar{y})=\frac{\partial x_{m}}{\partial y_{L}}(y, \bar{y})-\frac{\partial x_{l}}{\partial y_{L}}(y)
$$

Given that the rival is located at $\bar{y}$, we have $1 / 2 \leq x_{m}(y, \bar{y}) \leq x_{r}(y)$ for any $y \in[\underline{y}, \bar{y}]$. 
Claim 1. $\frac{\partial D_{L}}{\partial y_{L}}(y, \bar{y}) \leq \frac{\partial D_{1}}{\partial y}(y)$ for any $y \in(\underline{y}, 1 / 2)$.

This claim implies $\left|\frac{\partial D_{L}}{\partial y_{L}}(y, \bar{y})\right| \geq\left|\frac{\partial D_{1}}{\partial y}(y)\right|>0$ for $y \in(\underline{y}, 1 / 2)$. Hence, if the $L$ firm moves to the left in $(\underline{y}, 1 / 2)$, its indirect demand increases more than the increase in indirect demand experienced by the monopolist from the same change in $y$. Therefore, it is optimal for the former to choose $y$ when we consider $y \leq 1 / 2$.

Claim 2. $\frac{\partial D_{1}}{\partial y}(y) \geq \frac{\partial D_{L}}{\partial y}(y, \bar{y})$ for any $y \in(1 / 2, \bar{y})$.

This claim implies that if the $L$ firm moves to the right in $(1 / 2, \bar{y})$, its indirect demand increases less than the increase in indirect demand experienced by the monopolist from the same change in $y$.

As the monopolist's indirect demand is symmetric around $1 / 2$ and is maximized when it chooses $y=y$ or $y=\bar{y}$, Claim 1 and 2 imply that it is optimal for the $L$ firm to choose $\underline{y}$ (see Figure 4).

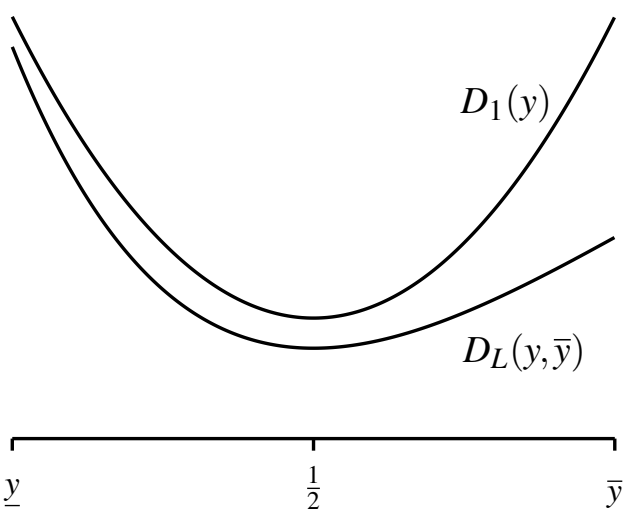

Figure 4: Indirect demand functions of the monopolist and the duopolist. The monopolist has a larger loss (respectively, gain) than the duopolist for $y<1 / 2$ (respectively, $y>1 / 2$ ).

Claim 1 is equivalent to $\frac{\partial x_{m}}{\partial y_{L}}(y, \bar{y}) \leq \frac{\partial x_{r}}{\partial y}(y)$ for $y \in(\underline{y}, 1 / 2)$ and Claim 2 is equivalent to $\frac{\partial x_{r}}{\partial y}(y) \geq \frac{\partial x_{m}}{\partial y_{L}}(y, \bar{y})$ for $y \in(1 / 2, \bar{y})$.

Hence, to prove both claims, it is enough to show $\frac{\partial x_{m}}{\partial y_{L}}(y, \bar{y}) \leq \frac{\partial x_{r}}{\partial y}(y)$, for any $y \in[\underline{y}, \bar{y}]$.

Now, we have

$$
\frac{\partial x_{m}}{\partial y_{L}}(y, \bar{y})=\frac{1}{2 \mu_{x}\left(x_{m}\right)}
$$

and

$$
\frac{\partial x_{r}}{\partial y}(y)=\frac{2\left(\mu\left(x_{r}\right)-y\right)}{2 \mu_{x}\left(x_{r}\right)\left(\mu\left(x_{r}\right)-y\right)+\sigma_{x}^{2}\left(x_{r}\right)}
$$


Therefore, $\partial x_{m} / \partial y_{L} \leq \partial x_{r} / \partial y$ if and only if

$$
2\left(2 \mu_{x}\left(x_{m}\right)-\mu_{x}\left(x_{r}\right)\right)\left(\mu\left(x_{r}\right)-y\right) \geq \sigma_{x}^{2}\left(x_{r}\right)
$$

1. In case we have $\mu$ convex on $[0,1 / 2]$ and $\sigma^{2}$ constant on $[0,1 / 2]$ :

From $\mu(x)$ convex on $[0,1 / 2]$, and from symmetry, follows that $\mu(x)$ is concave on $[1 / 2,1]$. Therefore, for any $y \in[\underline{y}, \bar{y}]$

$$
\mu_{x}\left(x_{m}(y, \bar{y})\right)>\mu_{x}\left(x_{r}(y)\right)
$$

which is equivalent to

$$
\frac{1}{\mu_{x}\left(x_{m}(y, \bar{y})\right)}<\frac{1}{\mu_{x}\left(x_{r}(y)\right)}
$$

Therefore,

$$
\frac{\partial x_{m}}{\partial y_{L}}(y, \bar{y})=\frac{1}{2 \mu_{x}\left(x_{m}(y, \bar{y})\right)}<\frac{1}{\mu_{x}\left(x_{m}(y, \bar{y})\right)}<\frac{1}{\mu_{x}\left(x_{r}(y)\right)}=\frac{\partial x_{r}}{\partial y}(y) .
$$

From the above argument, it is clear that $\partial x_{m} / \partial y_{L} \leq \partial x_{r} / \partial y$ holds when $\sigma_{x}^{2}<0$ on $[0,1 / 2]$ (or, equivalently, $\sigma_{x}^{2}>0$ on $[1 / 2,1]$ ) as well.

Furthermore, as we have some strict gap created by comparison between $\frac{1}{2 \mu_{x}\left(x_{m}\right)}$ and $\frac{1}{\mu_{x}\left(x_{r}\right)}$, the result carries out even if $\sigma_{x}^{2}>0$ but small on $[0,1 / 2]$.

2. In case we have $\mu$ linear and $\sigma^{2}$ concave on $[0,1 / 2]$ :

Let $\mu(x)=m x$, for $x \in[0,1 / 2)$. Hence, $\mu(x)=1-m(1-x)$, for $x \in(1 / 2,1]$.

In that case, $\frac{\partial x_{m}}{\partial y_{L}}(y, \bar{y}) \leq \frac{\partial x_{r}}{\partial y}(y)$ requires $\sigma_{x}^{2}\left(x_{r}\right) \leq 2 m\left(\mu\left(x_{r}\right)-y\right)$, that is, the slope of $\sigma^{2}$ cannot be very negative on $[0,1 / 2]$ (equivalently, not very positive on $[1 / 2,1]$ ).

Both in the case (i) $\sigma^{2}$ is linear and $\mu$ is either linear or concave and in the case (ii) $\mu$ is linear and $\sigma^{2}$ is convex, the limit polarization is not optimal in the baseline model without competition. Therefore, those cases are ruled out from our analysis.

\section{Proof of Proposition 7}

Let us start by showing that the optimum $y$ cannot be more extreme than $y^{*}$.

For $y \leq y^{*}$ we have

$$
D_{2}(y)=\int_{0}^{x_{r}(y)} \tilde{G}\left(x_{r}(y) ; x\right) d x
$$


Thus, as $x_{r}(y)$ is increasing in $y$, also $D_{2}(y)$ is increasing in $y$ for any $y \leq y^{*}$.

Suppose that the limit polarization is optimal in the one-layer maximization problem. Then, for any $y \in\left(y^{*}, 1 / 2\right]$, we have that $x_{r}(y)-x_{l}(y)<x_{r}\left(y^{*}\right)-x_{l}\left(y^{*}\right)=x_{r}\left(y^{*}\right)$. It is enough to verify that the limit polarization maximizes the second-layer indirect demand.

$$
D_{2}\left(y^{*}\right)=\int_{0}^{x_{r}\left(y^{*}\right)} \tilde{G}\left(x_{r}\left(y^{*}\right) ; x\right) d x=h_{1}\left(0, x_{r}\left(y^{*}\right)\right) .
$$

Thus, if homophily is extremal, for any $y \in\left(y^{*}, 1 / 2\right]$, we have

$$
D_{2}\left(y^{*}\right)=h_{2}\left(0, x_{r}\left(y^{*}\right)\right)>h_{2}\left(x_{l}(y), x_{r}\left(y^{*}\right)\right) .
$$

In addition, the following inequality holds

$$
h_{2}\left(x_{l}(y), x_{r}\left(y^{*}\right)\right) \geq D_{2}(y)=\int_{x_{l}(y)}^{x_{r}(y)} \tilde{G}\left(x_{r}(y) ; x\right)-\tilde{G}\left(x_{l}(y) ; x\right) d x
$$

since we have

$$
\begin{aligned}
h_{2}\left(x_{l}(y), x_{r}\left(y^{*}\right)\right)= & \int_{x_{l}(y)}^{x_{l}(y)+x_{r}\left(y^{*}\right)} \tilde{G}\left(x_{l}(y)+x_{r}\left(y^{*}\right) ; x\right)-\tilde{G}\left(x_{l}(y) ; x\right) d x \\
= & \int_{x_{r}(y)}^{x_{l}(y)+x_{r}\left(y^{*}\right)} \tilde{G}\left(x_{l}(y)+x_{r}\left(y^{*}\right) ; x\right)-\tilde{G}\left(x_{l}(y) ; x\right) d x \\
& +\int_{x_{l}(y)}^{x_{r}(y)} \tilde{G}\left(x_{l}(y)+x_{r}\left(y^{*}\right) ; x\right)-\tilde{G}\left(x_{r}(y) ; x\right) d x+D_{2}(y) \\
\geq & D_{2}(y)
\end{aligned}
$$

where the last inequality comes from the non-negativity of the terms with integrals. This proves that the limit polarization maximizes the second-layer indirect demand.

The proof for the case of no polarization is analogous.

\section{Proof of Proposition 8}

Let $t^{*}$ denote the depth of sharing the news located at $y$. Since, by assumption, a consumer located at $x$ reshares the news located at $y$ whenever both $U_{t(y)}(x, y) \geq 0$ and $B_{t^{*}}(x, y) \geq \tau$, if the attention tax is not small, the latter inequality is the first to bind. Therefore,

$$
\begin{aligned}
B_{t^{*}}(x, y) & =\int_{0}^{1} U_{t^{*}}(z, y) \tilde{g}(z ; x) d z \\
& =\delta^{t^{*}} u-\sigma^{2}(x)-(y-\mu(x))^{2} .
\end{aligned}
$$


And from $B_{t(y)}(x, y)=\tau$ follows that

$$
\delta^{t^{*}} u-\tau=\sigma^{2}(x)+(y-\mu(x))^{2} .
$$

As the L.H.S. of the equation above is decreasing in $t^{*}$, the depth is maximized when the R.H.S. is minimized. The result follows. 